The Journal of Engineering and Exact Sciences - jCEC, Vol. 06 N. 03 (2020)

journal homepage: https://periodicos.ufv.br/ojs/jcec

doi: 10.18540/jcecvl6iss3pp0206-0213

OPEN ACCESS - ISSN: 2527-1075

\title{
UTILIZACIÓN DEL MODELO DES, CON BASE EN EL MODELO DE TURBULENCIA SPALART-ALLMARAS EN EL ANÁLISIS DEL PERFIL S809
}

\section{USE OF THE DES MODEL BASED ON THE SPALART-ALLMARAS TURBULENCE MODEL IN THE S809 ANALYSIS OF AIRFOIL}

Pedro Casanova Treto ${ }^{1}$, Kattia Solís Ramírez ${ }^{2}$, Júlio Cesar Costa Campos $^{3}$, Luciano José Minette ${ }^{3}$, Antonio Marcos de Oliveira Siqueira ${ }^{4}$

1 Universidad de Costa Rica, Instituto de Investigaciones en Ingeniería (INII), San Pedro, Montes de Oca., Costa Rica 2 Universidad de Costa Rica, Escuela de Ingeniería de Biosistemas (EIB), San Pedro, Montes de Oca, Costa Rica 3 Universidade Federal de Viçosa, Departamento de Engenharia de Produção e Mecânica, Viçosa, Minas Gerais, Brasil 4 Universidade Federal de Viçosa, Departamento de Química, Viçosa, Minas Gerais, Brasil

\begin{tabular}{l} 
A R T I C L E I N F O \\
\hline Article history: \\
Received 2020-05-19 \\
Accepted 2020-09-04 \\
Available online 2020-09-04 \\
pa lav r a s - ch a ve \\
Spalart-Allmaras \\
S809 \\
Aerodinámica \\
Aerogenerador \\
Ke ywo rds \\
Spalart-Allmaras \\
S809 \\
Aerodynamics \\
Wind Turbine
\end{tabular}

\begin{abstract}
R E S U M N
Este trabajo brinda el estudio numérico de las características aerodinámicas del perfil S809 para un número de Reynolds de 300000. El perfil 5809 es uno de los perfiles más utilizados en aerogeneradores de baja potencia y bastante estudiado experimentalmente por varios autores. Para el estudio se utilizó el programa FLUENT del paquete ANSYS donde se seleccionó el modelo de turbulencia Spalart-Allmaras. Durante el estudio se utilizaron ángulos de ataque que variaron desde $0^{\circ}$ hasta $15,2^{\circ}$ determinándose los coeficientes de arrastre y sustentación para cada posición. El comportamiento aerodinámico numérico del perfil seleccionado presentó excelentes resultados cuando comparados con resultados experimentales reportados en la literatura. Se determinó que el modelo de turbulencia utilizado representa adecuadamente el proceso físico, pero a partir de un ángulo de ataque de $10,2^{\circ}$ comienza a desviarse levemente de los resultados experimentales, lo que puede ser explicado debido al aumento de la turbulencia por el aumento del ángulo de ataque
\end{abstract}




\section{INTRODUCCIÓN}

La turbulencia es un fenómeno caótico, es decir, un fenómeno complejo que está presente en los principales flujos prácticos de ingeniería, como el flujo en cavidades abiertas, burbujas, turbinas eólicas, aviones y combustión en general. Además, se estudian las turbulencias en la atmósfera y sus efectos en el vuelo de los aviones.

Es de conocimiento práctico y se evidencia en varias investigaciones y libros, que la transición a la turbulencia se caracteriza por la aparición de inestabilidades en un flujo, al principio laminar, que se multiplican por un proceso no lineal y degenerativa llamado flujo turbulento (NZEBUKA; WAHEED, 2020).

Se puede encontrar una revisión completa de RANS, DNS, LES reportada por Bellakhal et al. (2020), Cai et al. (2018), Deskos et al. (2020), Maragkos y Merci (2020), Ouyang et al. (2019), Ricci et al. (2020), Shi et al. (2020) y Xiao et al. (2020) mostrando sus ventajas y desventajas.

Además de estos modelos, existen otros modelos, llamados modelos de ecuaciones, que se utilizan ampliamente en la simulación de turbulencias, de acuerdo con Zhang et al. (2020). Algunos de estos modelos son el Spalart-Allmaras, Rahman-Agarwal-Siikonen, Wray-Agarwal y Xu-ZhangBaimodel (RAHMAN et al., 2011; SPALART; ALLMARAS, 1994; TANG et al., 2020; XU et al., 2016; ZHANG et al., 2020).

Los autores Shur et al. (1999) y Spalart et al. (2006) presentaron las primeras aplicaciones verdaderas de la simulación por DES (Detached-Eddy Simulation). El DES se definió en 1997 con la esperanza de combinar las fortalezas de los métodos de los promedios de Reynolds (RANS) y las simulaciones a gran escala, de manera no zonal, para tratar flujos separados con altos números de Reynolds. Los autores enfatizan la actividad de este modelo, inicialmente restringido al modelo de turbulencia de una ecuación, es decir, SpalartAllmaras. Sin embargo, según ellos, no existen restricciones para su uso en otros modelos. Además, se plantea que DES fue originalmente diseñado para tratar toda la capa límite utilizando un modelo RANS y para aplicar un tratamiento LES en regiones separadas.

Según Spalart (2009), la formulación DES original se basó en el modelo simple de Spalart-Allmaras, y ningún sistema CFD debe limitarse a un modelo. La motivación inicial para los altos números de Reynolds, así como para los flujos masivamente separados, permanece, para lo cual DES es más capaz hoy que los modelos RANS y LES.

En realidad, este modelo representa una combinación del modelo RANS y el modelo LES (HAN et al., 2017). La idea básica de DES es aplicar las ecuaciones del modelo RANS junto con el modelo de turbulencia en capa límite, donde LES tiene un alto costo computacional y usar LES en el resto del flujo.

Casadei et al. (2019) enfatizan la importancia de la dinámica de los fluidos computacionales en el estudio de los perfiles aerodinámicos, específicamente, en el sector aeroespacial. Este sector adopta herramientas computacionales para el estudio de la aerodinámica de los aviones, así como estimaciones de rendimiento. En este trabajo se prioriza el modelo DES en relación con RANS y LES validándolo con los datos del coeficiente y sustentación utilizando los modelos DES y RANS

El elemento básico de un aerogenerador es el rotor, que está formado por una o varias palas. El rotor es una de las partes fundamentales pues es quien captará la energía contenida en el fluido para su posterior transformación a energía eléctrica. Debido a esto es de vital importancia conocer el comportamiento aerodinámico de cada uno de los perfiles utilizados. Para esto se realizan pruebas de comportamiento aerodinámico tanto en túnel de viento como por medio de herramientas de CFD.

Autores como Butterfield et al. (1992), Castelli et al., (2012), Lee et al. (2012), Mirzaei et al., (2009), Wolfe y Ochs (1997) y Yao et al. (2012) han realizado estudios de diferentes perfiles, tanto experimentalmente como numéricamente. Debido a los reportes experimentales existentes es que se puede validar un modelo numérico que reproduzca con mayor exactitud el proceso físico. En este caso Butterfield et al. (1992) brindan los resultados experimentales para varios números de Reynolds del perfil S809. Es de vital importancia determinar el modelo que mejor se ajusta para cada una de las condiciones, pues permite realizar otros estudios que pueden ser a Reynolds inferiores donde existen escasos de resultados reportados.

Con el aumento de la velocidad de procesamiento de las computadoras y su capacidad de almacenamiento se torna posible realizar análisis numéricos de fenómenos físicos complejos. Debido a esto el estudio propone validar un modelo para el estudio del fenómeno físico que genere resultados próximos a la realidad y que puedan ser considerados seguros y precisos.

Otro aspecto de vital importancia es la turbulencia. Es necesario investigar los modelos de turbulencia más cercanos al fenómeno físico a estudiar, los cuáles van a estar en función del tipo de régimen de flujo bajo el cuál trabaja el aerogenerador. Además, el lugar del emplazamiento también definirá las intensidades de turbulencia a las cuales va a estar sometida la turbomáquina.

El principal objetivo analizado en este documento se refiera a la relación entre sustentación y arrastre. Cuanto mayor sea esta relación, más eficiente será el perfil en la transformación de la energía eólica en la energía eléctrica. Recientemente, existe una gran cantidad de estudios en todo el mundo en la búsqueda de la optimización del rendimiento aerodinámico del perfil al reducir el arrastre y aumentar la sustentación (SHI et al., 2020; YAO et al., 2012).

Otro punto importante en el estudio de perfiles aerodinámicos es la pérdida de sustentación o stall. Un perfil aerodinámico no se puede utilizar en todas las inclinaciones deseadas porque en algunos de ellos ocurrirá ese fenómeno. Stall es el nombre dado a la pérdida repentina de sustentación, que ocurre debido a la separación del fluido de la superficie del perfil, siendo uno de los principales impedimentos para aumentar la eficiencia de la turbina eólica (SHI et al., 2020). 


\section{MODELO DE TURBULENCIA Y ECUACIONES GOBERNANTES}

Spalart-Allmaras es un modelo de turbulencia representado por una ecuación de gobierno única y relativamente simple indicada por la Ecuación 2, que está modelada para la viscosidad cinemática (turbulenta). Este modelo ha sido diseñado específicamente para aplicaciones aeronáuticas y aeroespaciales que involucran flujos de pared limitados y ha mostrado buenos resultados para capas límite sujetas a gradientes de presión adversos. Además, está ganando popularidad para las aplicaciones de turbomaquinaria.

En el presente estudio, DES se basa en el modelo de Spalart y Allmaras (1994). Este modelo utiliza la ecuación de transporte para la viscosidad turbulenta modificada, $\hat{v}(\mathrm{HUH}$; LEE, 2018). El coeficiente de viscosidad de la turbulencia y la ecuación de transporte son:

$\mu_{\mathrm{t}}=\rho \hat{v} f_{v 1}$

$\frac{\mathrm{D} \hat{v}}{\mathrm{Dt}}=\mathrm{c}_{\mathrm{b} 1} \hat{S} \hat{v}-C_{\omega 1} \mathrm{f}_{\omega}\left(\frac{v}{d}\right)+\frac{1}{\sigma}\left[\nabla \cdot((v+\hat{v}) \nabla \hat{v})+\mathrm{c}_{\mathrm{b} 2}(\nabla \hat{v})^{2}\right]$

El término fuente y las funciones de amortiguación se definen como:

$$
\begin{array}{ll}
\hat{S}=\Omega+\frac{\hat{v}}{\kappa^{2} d^{2}} \mathrm{f}_{v 2}, & \mathrm{f}_{v 1}=\frac{\chi^{3}}{\chi^{3}+C_{v 1}^{3}}, \\
\mathrm{f}_{v 2}=1-\frac{\chi}{1+\chi \mathrm{f}_{v 1}}, & \chi=\frac{\hat{v}}{v}, \quad f_{\omega}=g\left[\frac{1+C_{\omega 3}^{6}}{g^{6}+C_{\omega 3}^{6}}\right]^{1 / 6}, \\
g=r+C_{\omega 2}\left(r^{6}-r\right), & r=\min \left[\frac{\hat{v}}{\hat{S} \kappa^{2} d^{2}}, 10\right],
\end{array}
$$

Donde $\rho$ es la densidad, $v$ es la viscosidad molecular, $\Omega$ es la vorticidad, $\mathrm{f}_{v 1}$ es la función de amortiguación viscosa, que se utiliza para garantizar que la viscosidad turbulenta esté bien predicha en la región afectada por la viscosidad y en las subcapas, y la distancia más cercana a la superficie de la pared. Los valores de las constantes del modelo están disponibles en Emami et al. (2020), Spalart y Allmaras (1994).

\section{METODOLOGÍA}

Este estudio fue realizado con el perfil S809 (Figura 1) el cual ha sido estudiado por varios autores lo que permite validar los resultados con mayor facilidad (BUTTERFIELD et al., 1992; RAMSAY et al., 1995; WOLFE; OCHS, 1997). El objetivo principal es estudiar dicho perfil para un número bajo de Reynolds, en este caso fue seleccionado 300000, donde Butterfield et al. (1992) brindan en su trabajo características aerodinámicas obtenidas en túnel de viento.

Para el análisis numérico primeramente se generó el perfil S809 y su correspondiente dominio de control en 2D mediante CAD. Posteriormente, se generó la malla de tipo estructurada en forma de C-H con la ayuda del programa ICEMCFD perteneciente a ANSYS (Figura 2), paquete comercial de CFD. La malla consta de 71400 elementos para un $\mathrm{y}+<10$ como se muestra en la Figura 3.

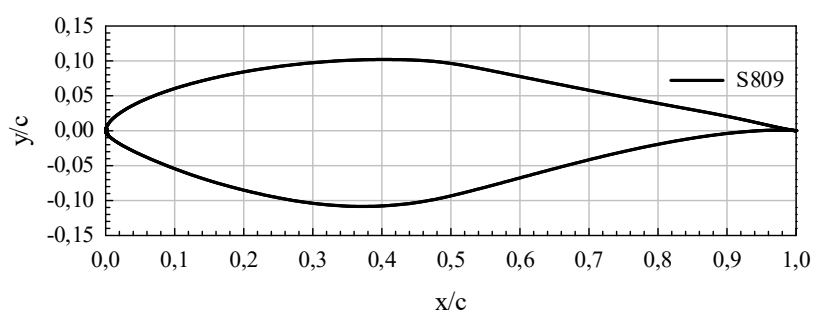

Figura 1- Representación del perfil S809.

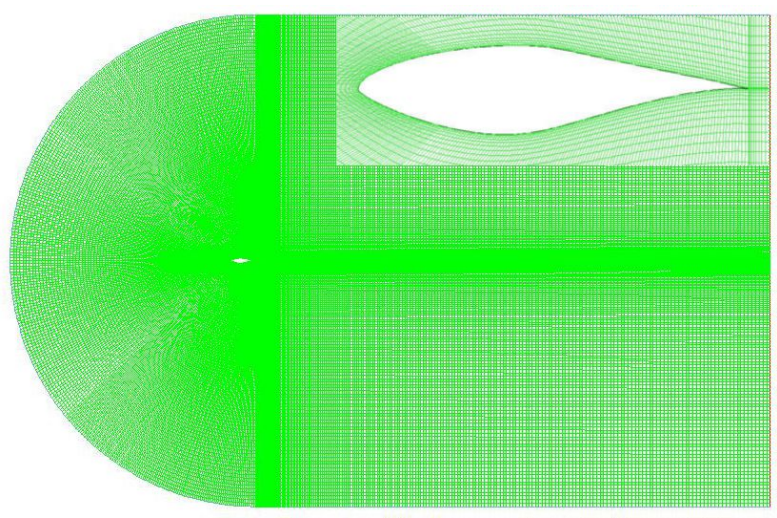

Figura 2- Malla estructurada tipo C-H utilizada.

Ribeiro et al. (2016) realizaron un estudio de malla estructurada aplicada al perfil S809. Utilizaron el generador de mallas ICEM del paquete ANSYS para generar la malla tipo C$\mathrm{H}$ para dicho perfil. El autor enfatiza que es una de las principales etapas de la simulación por computadora, ya que representará su dominio continuo a través de puntos interconectados, donde se resolverán las ecuaciones que rigen su fenómeno.

En este trabajo, un valor $\mathrm{y}+$ de aproximadamente $10 \mathrm{se}$ definió como el límite máximo, como se muestra en la Figura 3, siendo un buen compromiso entre los recursos computacionales disponibles y la precisión esperada.

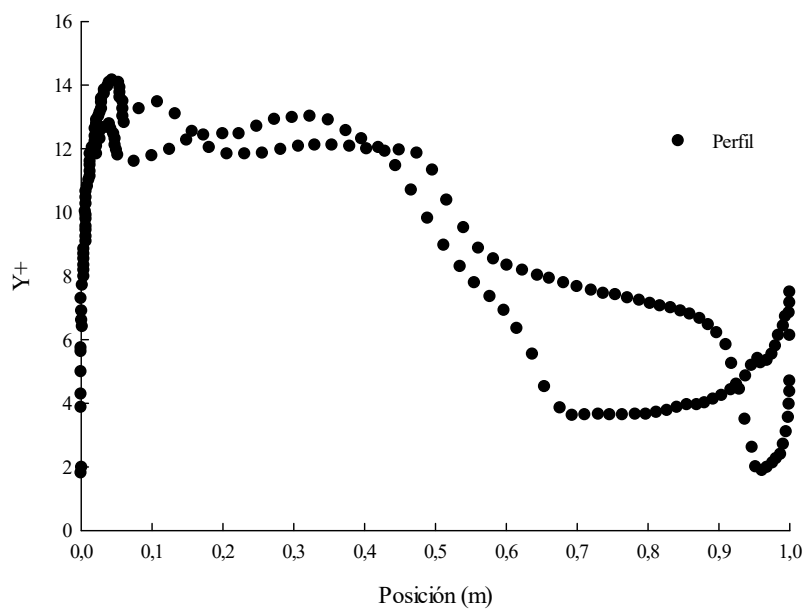

Figura 3- Distancia adimensional $\mathrm{y}^{+}$sobre la pared del perfil.

Según Casadei et al. (2019), la distancia adimensional desde la pared $\mathrm{y}^{+}$, es un parámetro que describe qué tan gruesa 
o delgada es la malla para un patrón de flujo específico. Un valor de $\mathrm{y}^{+}$cercano a 1 significa que la cuadrícula es lo suficientemente delgada como para representar toda la física del flujo, mientras que los valores más grandes indican que la malla es más gruesa de lo necesario para una resolución óptima.

El preprocesamiento y post-procesamiento fue realizado mediante el programa FLUENT, también perteneciente a ANSYS. Con dicho programa se utilizó el modelo de turbulencia Spalart-Allmaras, que brinda la posibilidad de utilizar menos tiempo computacional que otros modelos presentes en este programa brindando resultados similares. Cada una de las simulaciones fue realizada para un intervalo de ángulos de ataque entre $0^{\circ}$ y $15,2^{\circ}$. Las simulaciones fueron fijadas en un intervalo de 10000 iteraciones como máximo con residuos menores o iguales a $10^{-4}$.

\section{DISCUSIÓN DE RESULTADOS}

Debido a la acción del viento sobre el perfil, las fuerzas de presión actúan normal a la superficie de este, mientras los esfuerzos cortantes actúan de forma tangencial a la superficie del perfil; por tanto, integrando estas dos distribuciones sobre la superficie del perfil se tendrá una fuerza resultante y un momento. Esta fuerza resultante puede ser dividida en dos componentes: una perpendicular a la cuerda del perfil (fuerza normal) y la otra paralela a la cuerda del perfil (fuerza axial). Estas fuerzas pueden ser determinadas fácilmente durante el análisis de resultados en el FLUENT, el cual brinda a su vez los respectivos coeficientes axial $\left(\mathrm{C}_{\mathrm{A}}\right)$ y normal $\left(\mathrm{C}_{\mathrm{N}}\right)$ para cada uno de los ángulos de ataque $(\alpha)$ utilizados. Con esta información puede determinarse de manera sencilla los coeficientes de sustentación $(\mathrm{Cl})$ y arrastre $(\mathrm{Cd})$ como sigue:

$C_{l}=C_{N} \cos \alpha-C_{A} \operatorname{sen} \alpha$

$C_{d}=C_{N} \operatorname{sen} \alpha-C_{A} \cos \alpha$

La Figura 4 y 5 muestran los resultados de coeficiente de sustentación y arrastre obtenidos numéricamente.

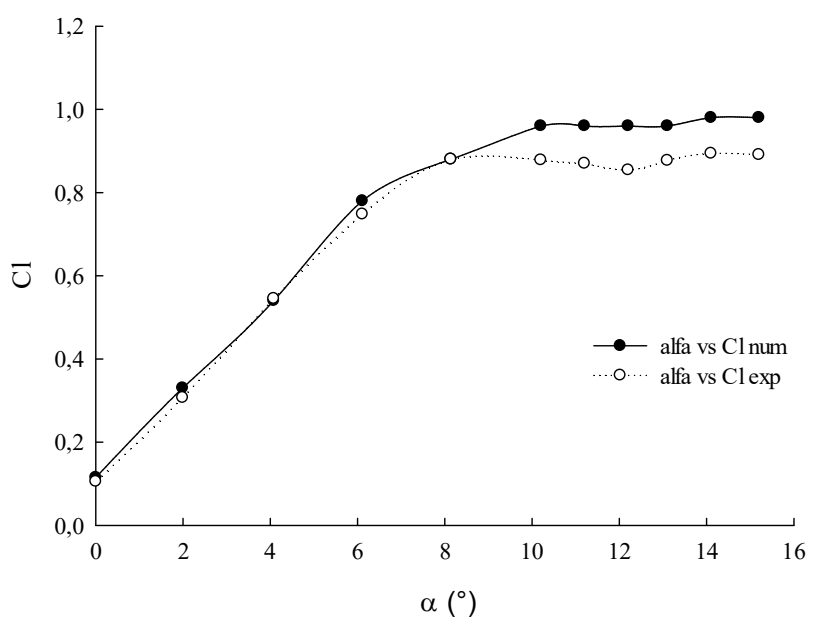

Figura 4- Comportamiento del coeficiente de sustentación para cada uno de los ángulos de ataque estudiados.

Puede observarse que el modelo de turbulencia SpalartAllmaras, utilizado para la obtención de los resultados reproduce aproximadamente el proceso físico en estudio.
Analizando los resultados obtenidos de coeficiente de sustentación (Figura 4), se puede determinar que hasta un valor de ángulo de ataque de $8,14^{\circ}$, el modelo reproduce fielmente el proceso comparándose con los resultados experimentales. Para ángulos superiores existe una pequeña discrepancia con los resultados apareciendo un ligero aumento de estos.

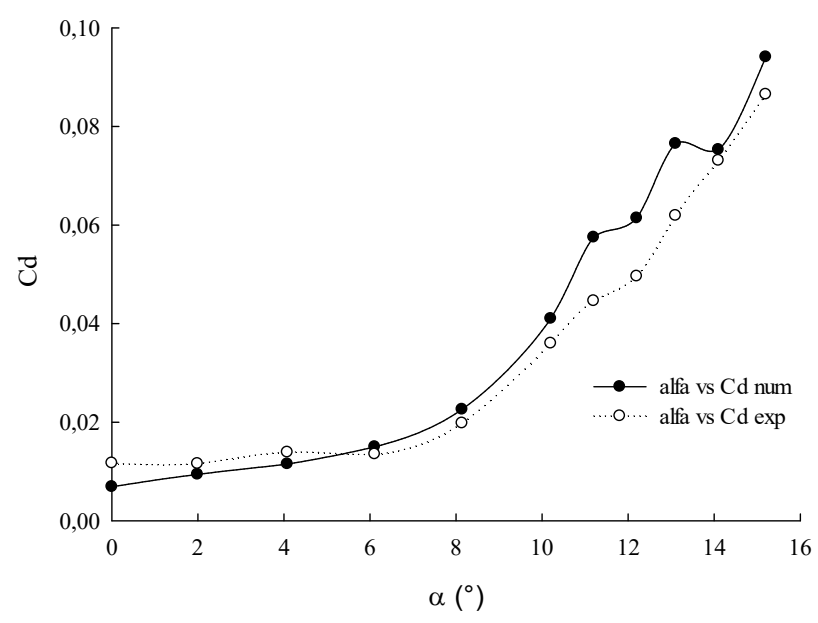

Figura 5- Comportamiento del coeficiente de arrastre para cada uno de los ángulos de ataque estudiados.

Comparando los coeficientes de arrastre (Figura 5) obtenidos con los experimentales puede determinarse que ocurre una situación similar a la reportada con el coeficiente de sustentación. Aunque para este coeficiente la discrepancia con los valores experimentales es para valores de ángulo de ataque superior a $10,2^{\circ}$.

Este comportamiento también se evidencia del mismo modo en trabajos de Castelli et al. (2012), Ramsay et al. (1995), Wolfe y Ochs (1997) y Yao et al. (2012). Debido a este patrón que se repite en diferentes trabajos consultados se evidencia la necesidad de estudiar de forma numérica con mayor profundidad el comportamiento aerodinámico de perfiles para altos ángulos de ataque.

En las Figuras 6 y 7 se muestran los contornos de velocidad y turbulencia para los ángulos de $0^{\circ}$ y $15,2^{\circ}$, que corresponden a los extremos del estudio realizado.

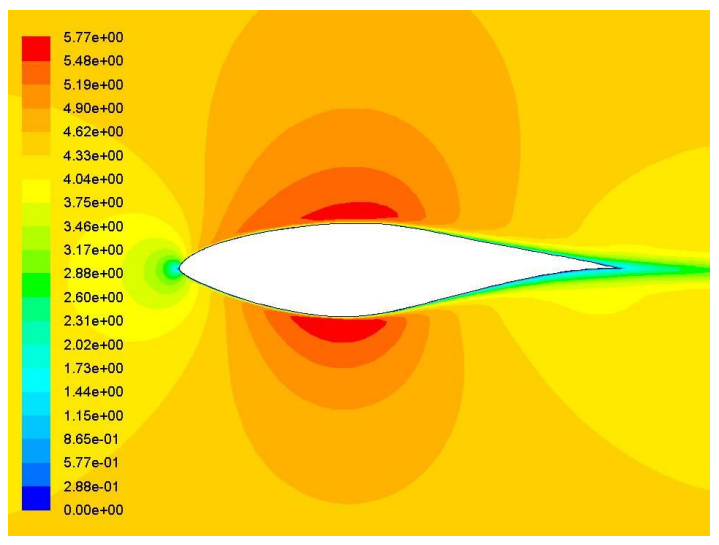

a) 

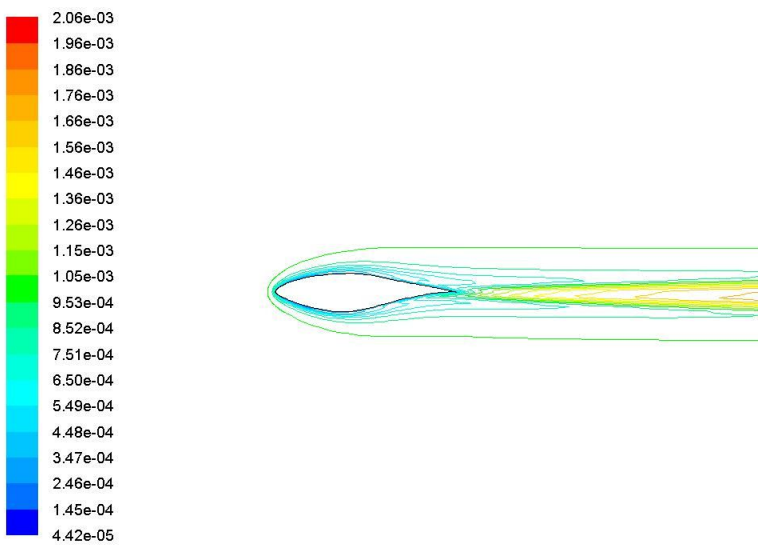

b)

Figura 6- a) Contornos de velocidad para ángulo de ataque de $0^{\circ}$; b) Contornos de turbulencia para ángulos de ataque de $0^{\circ}$.

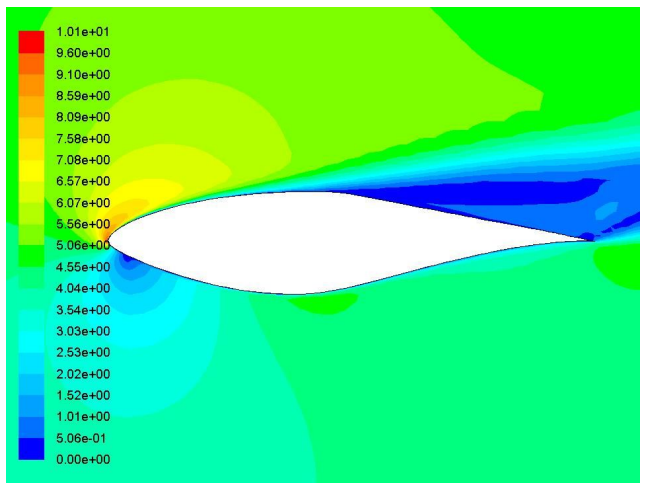

a)

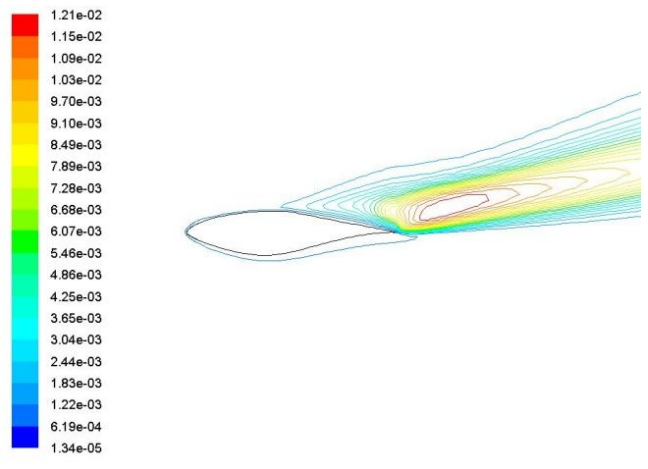

b)

Figura 7- a) Contornos de velocidad para ángulo de ataque de $15,2^{\circ}$; b) Contornos de turbulencia para ángulos de ataque de $15,2^{\circ}$.

En la Figura 6.a y 7.a se aprecia la presencia de dos regiones de transición que contienen burbujas de separación en la superficie superior e inferior del perfil S809. Este hallazgo fue enfatizado por Zhang et al. (2017) en su investigación sobre la simulación numérica en el flujo de transición para perfiles de aerogeneradores utilizando el modelo de turbulencia RANS. Estas cifras muestran la evolución de la capa límite, que es importante para comprender el proceso de transición. Además, con respecto a las burbujas de separación en la superficie del perfil S809, esto causa un déficit de velocidad.
Según Zhang et al. (2017), la burbuja de separación laminar es muy sensible a las perturbaciones del flujo y puede explotar durante la rotación de la pala. En consecuencia, puede causar el fenómeno de doble stall (pérdida de potencia), que disminuye significativamente el rendimiento de la turbina eólica (DEVINANT et al., 2002).

Devinant et al. (2002) destacan que, de hecho, si la transición en la parte superior de la burbuja de separación laminar y, por lo tanto, el reemplazo del flujo falla, la ruptura de la burbuja generalmente aparece, causando desprendimiento en el borde de ataque. Esto es muy sensible a pequeñas perturbaciones en el flujo y a menudo se observan flujos inestables asociados con la separación y el reemplazo del flujo, como el fenómeno de doble stall.

En las Figuras 6.b y 7.b, se puede apreciar la generación de vórtices en el borde de salida. En la Figura 6.b para un ángulo de ataque de $0^{\circ}$ donde, el desprendimiento de la capa límite todavía no está totalmente separado, se puede ver la formación de pequeños vórtices de amplitud en el borde de salida del perfil S809. Sin embargo, para un ángulo de ataque de $15,2^{\circ}$, en el borde de salida, se perciben vórtices de alta amplitud, formando una estela de Von Karman.

Puede apreciarse en ambos gráficos la influencia del ángulo de ataque sobre la turbulencia, donde ya para el ángulo de $15,2^{\circ}$ se incrementa la misma. Para el ángulo de $0^{\circ}$ prácticamente es nula la turbulencia debido a que el estudio se está realizando a número de Reynolds de 300000 que es una zona de transición donde es muy normal este resultado, pero ya al ir aumentando el ángulo de ataque se evidencia el aumento en la turbulencia.

Por tanto, esta pequeña desviación de los coeficientes de arrastre y sustentación puede estar dada debido al aumento en los valores de turbulencia por el incremento del ángulo de ataque. Estos resultados pudieran mejorarse realizando un estudio de la turbulencia para estos ángulos de ataque y calibrando el modelo utilizado en función del comportamiento de la turbulencia.

\section{CONCLUSIONES}

El modelo de turbulencia Spalart-Allmaras utilizado para la caracterización del fenómeno presentó excelentes resultados para ángulos de ataque inferiores a $10,2^{\circ}$, ya para valores superiores las diferencias entre lo numérico y lo experimental comienzan a aumentar.

La causa fundamental por la que comienzan a diferir los valores de coeficiente de arrastre y sustentación numéricos y experimentales son debidos al aumento del ángulo de ataque que provoca un aumento de la turbulencia.

El modelo utilizado se ajusta de manera adecuada al proceso físico y se recomienda realizar ajustes en el mismo para ángulos mayores a $10,2^{\circ}$ para incrementar su exactitud.

\section{AGRADECIMIENTOS}

Los autores agradecen a la UCR - Universidad de Costa Rica; CAPES - Coordenação de Aperfeiçoamento de Pessoal de 
Nível Superior; CNPq - Conselho Nacional de Desenvolvimento Científico e Tecnológico; FAPEMIG - Fundação de Amparo à Pesquisa do Estado de Minas Gerais; DEP - Departamento de Engenharia de Produção e Mecânica; UFV - Universidade Federal de Viçosa.

\section{REFERENCIAS}

BELLAKHAL, G.; CHAIBINA, F.; CHAHED, J. Assessment of turbulence models for bubbly flows: Toward a fiveequation turbulence model. Chemical Engineering Science, v. 220, 2020.

BUTTERFIELD, C. P.; MUSIAL, W. P.; SIMMS, D. A. Combined Experiment Final Report- Phase I.NREL/TP-257-4655. Golden, Colorado: 1992.

CAI, Z.; SUN, M.; WANG, Z.; BAI, X. S . Effect of cavity geometry on fuel transport and mixing processes in a scramjet combustor. Aerospace Science and Technology, v. 80, p. 309-314, 2018.

CASADEI, L.; KÖNÖZSY, L.; LAWSON, N. J. Unsteady Detached-Eddy Simulation (DES) of the Jetstream 31 aircraft in One Engine Inoperative (OEI) condition with propeller modelling. Aerospace Science and Technology, v. 91, p. 287-300, 2019.

CASTELLI, M. R.; GRANDI, G.; BENINI, E. Numerical analysis of laminar to turbulent transition on the DU91W2-250 airfoil. International Scholarly and Scientific Research \& Innovation, v. 6, n. 3, p. 717-724, 2012.

DESKOS, G.; del CARRE, A.; PALACIOS, R. Assessment of low-altitude atmospheric turbulence models for aircraft aeroelasticity. Journal of Fluids and Structures, v. 95, 2020 .

DEVINANT, P.; LAVERNE, T.; HUREAU, J. Experimental study of wind-turbine airfoil aerodynamics in high turbulence. Journal of Wind Engineering and Industrial Aerodynamics, v. 90, n. 6, p. 689-707, 2002.

EMAMI, S. M. K.; MOUSAVI, S. F.; HOSSEINI, K.; FOULADFAR, H.; MOHAMMADIAN, M Comparison of different turbulence models in predicting cohesive fluid mud gravity current propagation. International Journal of Sediment Research, 2020.

HAN, X.; WRAY, T. J.; AGARWAL, R. K. Application of a new DES model based on Wray-Agarwal turbulence model for simulation of wall-bounded flows with separation. In: 47th AIAA Fluid Dynamics Conference, 2017. Denver, Colorado: American Institute of Aeronautics and Astronautics Inc, AIAA, 2017.

HUH, J.; LEE, S. Numerical study on lateral jet interaction in supersonic crossflows. Aerospace Science and Technology, v. 80, p. 315-328, 2018.

LEE, S. G.; PARK, K. S.; LEE, K. S.; CHUNG, C. Performance prediction of NREL (National Renewable Energy Laboratory) Phase VI blade adopting blunt trailing edge airfoil. Energy, v. 47, n. 1, p. 47-61, 2012.

MARAGKOS, G.; MERCI, B. On the use of dynamic turbulence modelling in fire applications. Combustion and Flame, v. 216, p. 9-23, 1 jun. 2020.

MIRZAEI, M.; ARDEKANI, M. A.; DOOSTTALAB, M. Numerical and experimental study of flow field characteristics of an iced airfoil. Aerospace Science and Technology, v. 13, n. 6, p. 267-276, 2009.

NZEBUKA, G. C.; WAHEED, M. A. Thermal evolution in the direct chill casting of an Al-4 pet $\mathrm{Cu}$ alloy using the lowReynolds number turbulence model. International Journal of Thermal Sciences, v. 147, 2020.

OUYANG, Y.; TANG, K. L.; XIANG, Y.; ZOU, H. K.; CHU, G. W.; AGARWAL, R. K.; CHEN, J. F. Evaluation of various turbulence models for numerical simulation of a multiphase system in a rotating packed bed. Computers and Fluids, v. 194, 2019.

RAHMAN, M. M.; SIIKONEN, T.; AGARWAL, R. K. Improved low-reynolds-number one-equation turbulence model. AIAA Journal, v. 49, n. 4, p. 735-747, 2011.

RAMSAY, R. R.; HOFFMANN, M. J.; GREGOREK, G. M. Effects of grit roughness and pitch oscillations on the NACA 4415 airfoil. Nrel/Tp-442-7817. Golden, Colorado: 1995 .

RIBEIRO, W. M.; CAMPOS, J. C. C.; TIBIRIÇA, Á. M. B.; ROSA, H. M. P.; BRITO, R. F.; CASANOVA, P. T. . Utilização de uma malha estruturada na análise do aerofólio S809. In: BORGES, R. A.; QUEIROZ, T. A. (Org.). Matemática aplicada à indústria: problemas e métodos de solução. São Paulo: Blucher, 2016.

RICCI, A.; KALKMAN, I.; BLOCKEN, B.; BURLANDO, M.; REPETTO, M. P. Impact of turbulence models and roughness height in 3D steady RANS simulations of wind flow in an urban environment. Building and Environment, v. 171, 2020.

SHI, L.; WANG, Y.; ZHANG, G.; JIN, Y.; ZHANG, D. Assessment of an improved turbulence model in simulating the unsteady flows around a D-shaped cylinder and an open cavity. Applied Mathematical Modelling, v. 83, p. 552-575, 2020.

SHUR, M.; SPALART, P. R.; STRELETS, M.; TRAVIN, A. Detached-eddy simulation of an airfoil at high angle of attack. In: RODI, W.; LAURENCE, D. (Eds.). . Engineering Turbulence Modelling and Experiments 4. [s.1.] Elsevier Science Ltd, 1999. p. 669-678.

SPALART, P. R.; DECK, S.; SHUR, M. L.; SQUIRES, K. D.; STRELETS, M. K.; TRAVIN, A. A new version of detached-eddy simulation, resistant to ambiguous grid densities. Theoretical and Computational Fluid Dynamics, v. 20, n. 3, p. 181-195, 2006.

SPALART, P. R. Detached-Eddy Simulation. Annual Review of Fluid Mechanics, v. 41, n. 1, p. 181-202, 2009.

SPALART, P. R.; ALLMARAS, S. R. One-equation turbulence model for aerodynamic flows. Recherche aerospatiale, n. 1, p. 5-21, 1994.

TANG, K.L.; OUYANG, Y.; AGARWAL, R. K.; CHEN, J. M.; XIANG, Y.; CHEN, J. F. Computation of gas-liquid flow in a square bubble column with Wray-Agarwal oneequation turbulence model. Chemical Engineering Science, v. 218, 2020. 
WOLFE, W. P.; OCHS, S. S. CFD calculations of S809 aerodynamic characteristics. In: 35th Aerospace Sciences Meeting and Exhibit. Reston, Virginia: American Institute of Aeronautics and Astronautics, 1997.

XIAO, H.; WU, J. L.; LAIZET, S.; DUAN, L. Flows over periodic hills of parameterized geometries: A dataset for data-driven turbulence modeling from direct simulations. Computers and Fluids, v. 200, 2020.

XU, J. L.; SONG, Y. F.; ZHANG, Y.; JI, S. C.; BAI, J. Q. A turbulence characteristic length scale for compressible flows. Journal of Turbulence, v. 17, n. 9, p. 900-911, 2016.

YAO, J.; YUAN, W.; WANG, J.; XIE, J.; ZHOU, H.; PENG, M.; SUN, Y. Numerical simulation of aerodynamic performance for two dimensional wind turbine airfoils. Procedia Engineering, v. 31, p. 80-86, 2012.

ZHANG, Y.; SUN, Z.; van ZUIJLEN, A.; van BUSSEL, G. Numerical simulation of transitional flow on a wind turbine airfoil with RANS-based transition model. Journal of Turbulence, v. 18, n. 9, p. 879-898, 2017.

ZHANG, Y.; RAHMAN, M. M.; CHEN, G. Development of k$\mathrm{R}$ turbulence model for wall-bounded flows. Aerospace Science and Technology, v. 98, 2020. 